\title{
Up-link Performance of the DQRUMA MAC Protocol in a Realistic Indoor Environment for W-ATM Networks
}

\author{
Ch.V.Verikoukis, J.J.Olmos \\ Technical University of Catalunia (U.P.C.) \\ Signal Theory and Communications Department \\ Campus Nord-D4, Jordi Girona 1-3, 08034-Barcelona, Spain \\ e-mail:[cveri, olmos]@xaloc.upc.es
}

\begin{abstract}
This paper evaluates by simulation the performance of the Distributed Queuing Request Update Multiple Access (DQRUMA) MAC protocol in a realistic indoor environment. This protocol has been simulated in conjunction with a type-II Hybrid-ARQ protocol based on punctured R-S codes for the LLC layer. The analysis was carried out for a multicarrier modulation scheme (OFDM) with QPSK modulation on each carrier. The Hidden Markov Model (HMM) is used for modelling the physical layer of the system.

The performance of the protocol in terms of throughput, mean delay, CLR and p.d.f. of the delay is presented. Pseudo-bayesian algorithm as well as harmonic back-off are applied to calculate the retransmission probability for the backlogged users.
\end{abstract}

\section{Introduction}

To extend the capabilities of ATM over the wireless channel, the concept of Wireless ATM (W-ATM) was proposed in 1994 [1]. W-ATM is a promising technology for the future broadband services due to its capacity of providing different communication services, in various application environments, while guaranteeing a previously agreed quality of service (QoS).

A typical W-ATM network follows a protocol layer harmonised with the standard ATM. The wireless air interface consists of the Wireless Physical layer (W-PHY) and Data Link Control (DLC) layer. This last contains a Medium Access Control (MAC) and a Logical Link Control (LLC) sublayers. The MAC sublayer is in charge of implementing the multiple access scheme of W-ATM

This work was supported by CICYT project TIC 98-684 system, while the LLC sublayer performs a service class specific error correction scheme in order to guarantee high transmission quality.

In this paper the performance of the DQRUMA [2] protocol under realistic channel conditions for W-ATM networks is presented. DQRUMA is an efficient demandassign type channel access protocol which is designed for fixed-length packets considering a time slotted system with no frame reference. The study is focused in the uplink since, due to the limited power of the mobile transmitter, it is the worst conditioned of the two links.

The DQRUMA has been simulated in conjunction with a type-II Hybrid ARQ/FEC protocol based on punctured R-S codes [3] for the W-LLC and an OFDM system for the physical layer in an indoor environment. The physical layer is modelled by a HMM [4].

The remainder of the paper is structured as follows. In section 2 the physical layer is presented while in section 3 a brief description of the FMM is done. Section 4 explains the DQRUMA protocol with ideal channel whereas section 5 presents the DQRUMA with non-ideal channel. Section 6 analyses the pseudo-bayesian algorithm as well as the harmonic back-off. Finally the simulation model is described and simulation results are presented.

\section{Physical layer Description}

The physical layer provides a $20 \mathrm{Mb} / \mathrm{s}$ (uncoded) wireless link for ATM traffic in a radio link bandwidth of $25 \mathrm{MHz}$ and a carrier frequency of $5.2 \mathrm{GHz}$.

Orthogonal Frequency Division Multiplexing (OFDM) with 16 sub-carriers and QPSK modulation on each one has been selected as the modulation scheme. An Inverse Fast Fourrier Transformation (IFFT) and an oversampling factor of 4 is used to generate the time domain samples transmitted during one OFDM symbol. Oversampling is necessary to avoid aliasing in the generated signal 
spectrum. To add the cyclic prefix, some samples at the end of each OFDM symbol are replicated to the beginning of the symbol. This is used to protect the symbol from the echoes of the channel. The use of a time domain raised cosine (roll-off $=0.5$ ) windowing of each OFDM symbol is necessary to reduce the adjacent channel interference. The raised cosine window lasts $15.6 \%$ of the OFDM symbol at the beginning and at the end of each symbol. Thus the total symbol is made up of the windowing time, a FFT period of $1.28 \mu$ s and a $160 \mathrm{~ns}$ guard time (cyclic period). After modulation the signal is up-converted to the RF channel band.

Before the channel, the signal is power amplified using a non linear amplifier that can be characterised using the model of [5]. To limit the distortion due to the power amplifier without reducing too much its efficiency, a $3 \mathrm{~dB}$ back-off in the input signal is applied.

A three-ray, time-varying radio channel in a picocellular environment has been chosen for simulation. The power delay profile is based on the model of the COST 207-project [6].

At the receiver, a coherent QPSK demodulation takes place on every carrier, and hard decision bit by bit outputs are delivered. Pilots symbols for the channel transfer function estimation are used. This allows Maximal Ratio Combining diversity with two antennas. Ideal time and frequency synchronism is assumed.

In an OFDM based physical layer, the errors in the real system appear surrounding the carrier where the fading is centred. Therefore, a periodicity equal to the number of bits in an OFDM packet is observed in the errors position.

Since the R-S codes that we use are not binary, it is better to have short error clusters than isolated errors. The interleaving technique guarantees that all the bits of every code symbol travel in the same OFDM carrier. In this way, when there appear frequency selective fading events that produce errors only in few of the total number of carriers, the R-S decoder will see the errors as clustered in one or two code symbols and will easily correct them. This kind of interleaving exploits the inherent frequency diversity in the coded OFDM modulation and, since it is an intra-ATM cell reordering of the QPSK symbols, it does not increase the overall transfer delay.

\section{Hidden Markov Model}

Due to the long coherence time of the picocellular mobile channel, the detailed physical layer simulation requires programs that execute too slowly. To overcome this problem, the physical layer is modelled by a HMM. This method reproduces the statistical behaviour of the physical layer, in terms of error distribution.

To obtain the Markov model of the physical layer, a packet of 512 bit (equivalent to a coded ATM cell) is considered. Next, and $E_{b} / N_{0}$ values in steps of $1 \mathrm{~dB}$, we define 8 or 16 possible states of the physical layer. Each state represents a range of the number of errors in a packet. The range of number of errors assigned to every state should lead to states that are as equiprobable as possible. Using detailed physical layer simulations we obtain, for every state, the probabilities of passing from it to any one of the others. These probabilities form the "transition matrix", which is stored in a file for further processing.

For running the HMM it is sufficient to generate random numbers distributed in accordance to the transition matrix. In this way the simulated system passes from one state to the other with the right probabilities. Depending on the actual state corresponding to each transmitted packet, the exact number of errors is calculated according to the algorithm proposed in [7]. In [7] the errors are distributed inside the packet with uniform statistics. This is not adequate for OFDM based physical layers because as already mentioned, the errors appear surrounding the carrier where the fading is centered. Our method consists in obtaining, form the detailed simulations, the p.d.f. of the error distribution in the packets conditioned to the fact that the fading is centered in one of the carriers. This is done for every carrier. Then, when running the model, the errors are distributed inside the packet according to one, randomly selected, stored p.d.f.'s.

Although the fading in the real system remains in the same carrier for several packet intervals, we do not need to reproduce exactly this channel memory since it does not affect to the error correcting capacity of the R-S codes.

\section{DQRUMA description}

The DQRUMA is divided into two phases, a Request Access (RA) phase and a packet transmission phase. For the uplink (UL), each time slot is divided into Request Access (RA) channel and Cell Transmission (Xmt) channel. PGBK (Piggyback) Request information is also provided in the UL. For the Downlink (DL), each time slot is divided into ACK channel, Transmission Permission (Xmt perm) channel and Xmt-channel

When a cell arrives at an empty buffer, the mobile terminal (MT) sends a request (Xmt-Req) to the Base Station (BS) via the UL RA-channel. When the BS successfully receives a Xmt-Req from a MT, it sets the corresponding entry in the Request Table (RT) to indicate that a MT has a packet to transmit. The BS informs the reception of the Xmt-Req by broadcasting the Access ID over the DL. When NACK is received, the MT is dropped 


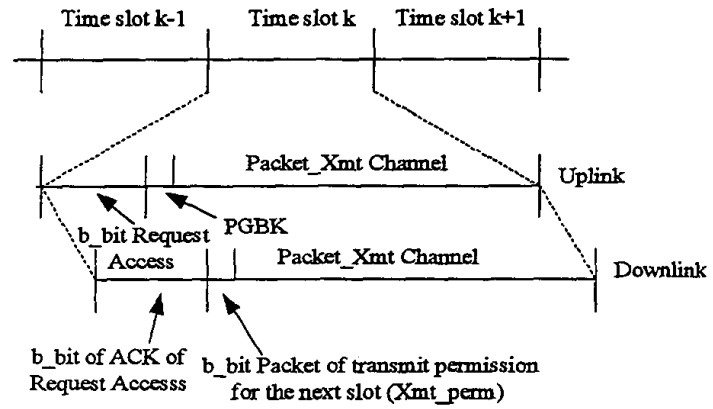

Figure 1 DQRUMA timing diagram

in backlog mode and tries to send a RA after a back-off interval

According to a desired packet transmission policy the BS chooses one of the MT that has a non empty Xmt-Req field in the RT and broadcasts the Access ID over the DL channel. This means that the MT will transmit to the next slot. With cell transmission is also includes PGBK Request, without contention, to indicate it has more packets in its buffer.

If there is no any other packet in the MT buffer, this MT is deleted from the RT. If all the Xmt-Request fields in the RT are empty, the BS uses the DL Xmt-Perm channel to announce that the next UL Xmt-channel will be converted into multiple RA-channels. In this protocol collision happens only on the RA channel. Therefore, contention for requests according to random access protocol, e.g. S-Aloha, is reduced and the system throughput under heavy load is greatly improved.

\section{DQRUMA under real channel conditions}

When non ideal channel is considered, in the RA phase only error detection is applied. A NACK message is sent to the MT if at least an error is detected by the CRC code in the Xmt-Req packet. Then the MT is dropped in backlog mode.

In the event of a collision, it may still be possible that a MT with the strongest signal strength captures the system.

When a MT has permission to transmit, the type-II Hybrid ARQ/FEC is applied in order to ensure reliable transmission under the worst channel conditions. In this case, when the error correcting capability of $C_{1}(n / 2, k)$ is not sufficient, a transmitter receives a NACK message. The erroneous code word is stored in the receiver buffer, the MT entry remains in the RT and the MT waits to hear its Access ID in the DL channel in order to transmit the associated $C_{2}$ code word. When $C_{2}$ is transmitted, the receiver joins together the two code words to form a corrupted version of a code word in $C_{3}(n, k)$. If the decoding operation does not succeed, a NACK message is received by the MT. The received code word from $C_{2}$ is kept in a receiver buffer and the above procedure is repeated. In case of a correct reception, if there is no any other packet in the MT buffer, the BS delete the corresponding entry from the RT. The flow chart of this procedure is shown in Figure 2.

A cell is discarded when it remains in the MT buffer, longer than the cell lifetime.

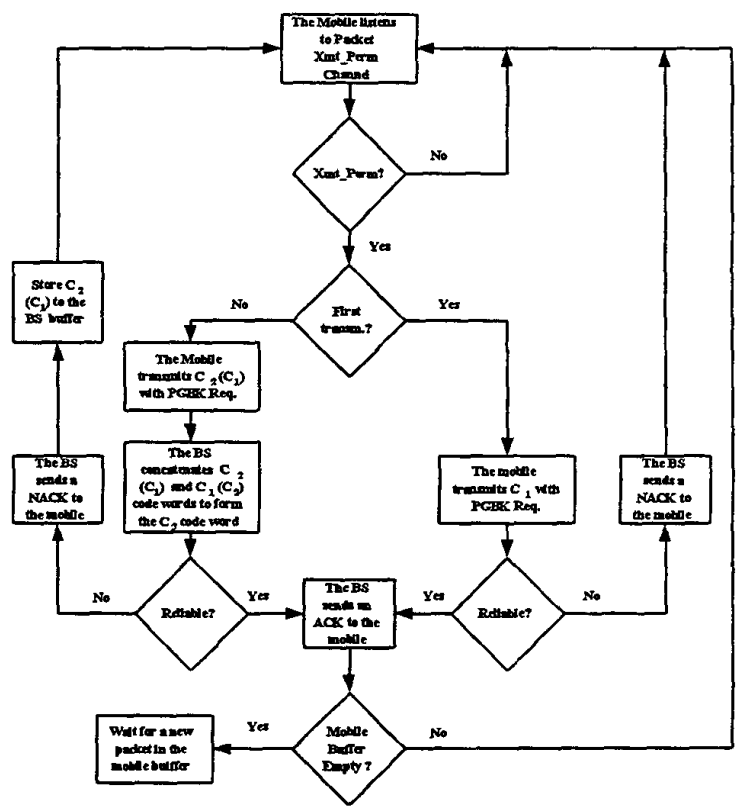

Figure 2 Transmission phase of the DQRUMA under real channel conditions

\section{Back-off probability}

\subsection{Pseudo-bayesian algorithm}

It is known that the basic slotted ALOHA algorithm, where a MT retransmits backlogged packets with fixed probability, is unstable.

The pseudo-bayesian algorithm [8] is a simple and effective way to stabilise ALOHA estimated the backlogged packets from the feedback.

In this algorithm the new arrivals are immediately regarded as backlogged. Rather than transmitted in the same way as packets involved in previous collisions.

Within the DQRUMA this algorithm operates by keeping and updating an estimation $n$ est of the number of backlogged users. The estimated backlog at the beginning of its slots $k$ is updated from the estimated backlog and feedback for slot $k-l$ according to the rule: 
$n_{-} e s t_{k}=\left\{\begin{array}{lc}\max \left\{\lambda, n_{-} e s t_{k-1}+\lambda-1\right\} & \text { for idle or } \\ n_{-} e s t_{k-1}+\lambda+(e-2)^{-1} & \text { for collision }\end{array}\right.$

According to [9] if the fixed value $1 / \mathrm{e}$ for the arrival rate $\lambda$ is used within the algorithm, stability is achieved for all actual $\lambda<1 / \mathrm{e}$.

Each backlogged user is then independently sends a $\mathrm{RA}$ to the BS in a slot $k$ with probability:

$$
p_{k}=\min \left(1,1 / n_{-} \text {est } t_{k}\right)
$$

\subsection{Harmonic Back-off}

When the harmonic-back is applied the RA probability of each user is first initialised to one. When a MT drops in backlog mode in the time slot $k-1$, it sends a RA in the time slot $k$ with probability:

$$
p_{k}(i)=\frac{p_{k-1}(i)}{p_{k-1}(i)+1}
$$

\section{Simulation Model}

In the simulation model, that has been written in $\mathrm{C}++$, the BS is located at the centre of a circular cell of unit radius and the position of the MTs is uniformly distributed in the circle. Before starting the simulation, the normalised, to the signal power at the border of the cell, received signal power of each $\mathrm{MT}$ is calculated and the corresponding transition matrix is assigned. The signal power is inversely proportional to the distance of the BS where path loss exponent has the typical for picocellular indoor environment value of 2 .

At the beginning of each time slot, the state of each transition matrix is updated. Using these transition matrices, the error distribution both in Xmt-Req packets and in transmitted cells are produced. Apart from these matrices, a set of transition matrices called "capture matrices" for values of $E_{b} / N_{0}$, in steps of $1 \mathrm{~dB}$, are also updated at the beginning of each slot. In case of a collision event, for the user with the higher power, a "capture power", depending on the power of the other collided users is calculated. Based on this value the appropriate transition matrix is used to produce the error distribution in the Xmt-Req packet.

Traffic model based on Bemoulli trials with average cell arrival rate $p$, in cells per time slot, for the source is used. In each time slot, the source selects a random number from $(0,1)$. This number is compared to a fixed value, $p$, in the same range. A cell is generated by the source in this slot if the random number is less than $p$.

Considering that our physical layer is OFDM based, each time slot consists of 17 OFDM blocks. The first of them is used as Xmt-Req packet. In the other 16 OFDM blocks the ATM payload, the ATM compressed header, all the other necessary headers, error detection and error correction bits are carried. Thus, an uplink can be converted into $17 \mathrm{RA}$-channels when there is no traffic.

\section{Simulations Results}

As a figure of merit to evaluate the performance of the DQRUMA, using Round Robin scheduling policy, plots for the mean delay and the Cell Loss Ratio (CLR) versus throughput are presented. The p.d.f of the system delay as well as the p.d.f. of the delay for a user situated in region close to the BS (user a), close to cell border (user $c$ ) and in an intermediate region (user $b$ ) are also evaluated.

The mean delay is measured in terms of time slots of 17 OFDM blocks (2.8305e-5 s), while the CLR is the ratio of the total lost cells to the total number of transmitted cells. As throughput the ratio of the number of received with success cells to the total number of slots is defined. In the throughput the time spent it in each time slot for the Xmt-RA packet is included.

A cell that remains to the MT buffer longer than $10 \mathrm{~ms}$ is discarded while minimum $E_{b} / N_{0}$ of $12 \mathrm{~dB}$ has been calculated at the cell border.

When capture phenomenon is applied in the RA phase, the efficiency in terms of mean delay is improved as in Figure 3 is shown. In this plot 50 users, harmonic backoff and ideal transmission are assumed. In the same figure the degradation due to the non ideal channel in the transmission phase is also shown.

Figure 4 allows to compare the efficiency of the pseudo-bayesian in terms of CLR with the harmonic backoff. Non ideal channel in the transmission phase is assumed. It is evident that when the pseudo-bayesian is used the CLR is lower.

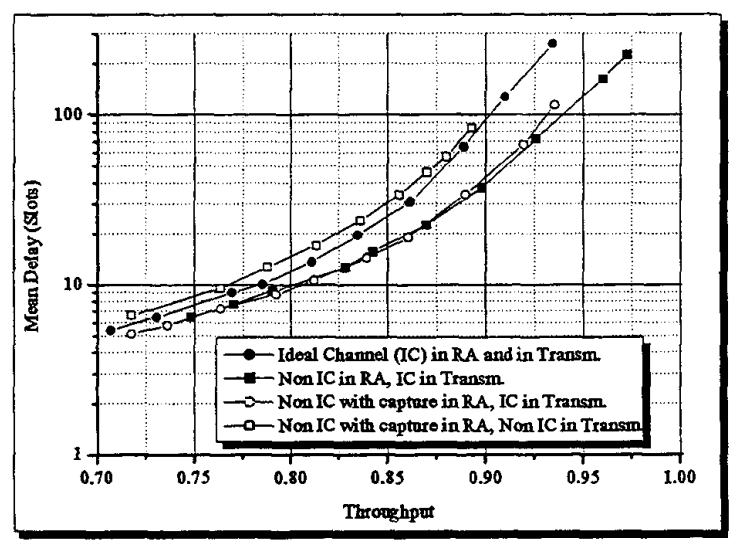

Figure 3 Mean delay in slots vs. throughput 


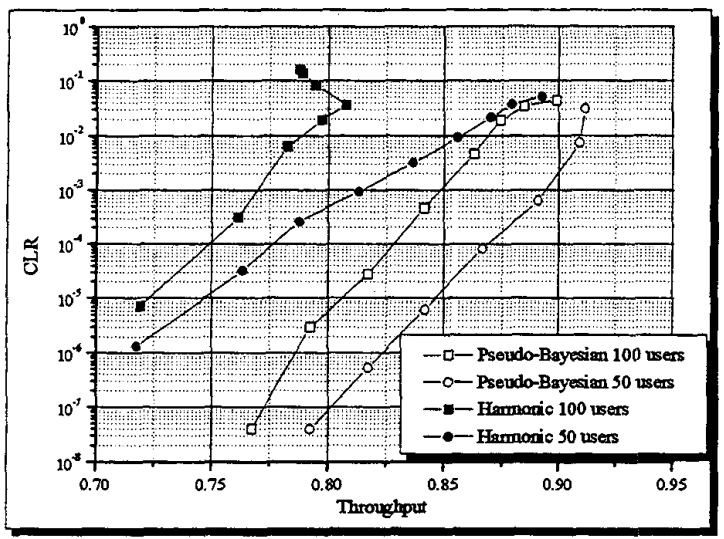

Figure 4 Cell Loss Ration vs. throughput

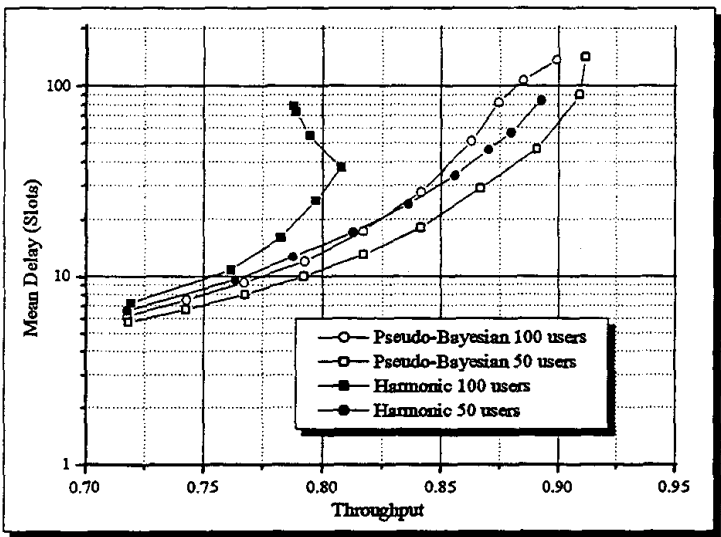

Figure 5 Mean delay vs. throughput

In terms of mean delay, as in Figure 5 is shown, the system performs also better in case of the pseudobayesian Furthermore, for 100 users the system does not collapse due to the overload.

Figure 6 presents the p.d.f. of the delay considering 50 users and a throughput value of 0.81 . Even though for this throughput value the mean delay with the pseudobayesian is slightly lower, high delay transmission events are hundred times less probable than those with harmonic back-off. The same result can be observed from the figures 7,8 and 9. Furthermore, we can observe that as faraway a MT located from the BS, high delays in the transmission appear frequently with harmonic back-off.

Finally the p.d.f. of the delay with the pseudo-bayesian for several throughput values is presented in Figure 10.

When the harmonic back-off is applied, the RA probability is calculated independently for each MT and it takes very low values after several backlog events. In case of the pseudo-bayesian the probability is calculated in each time slot using information from the feedback and it is the same for all the MTs. Therefore, the MTs have more probabilities to send a RA and consequently to get permission from the BS to deliver a cell.

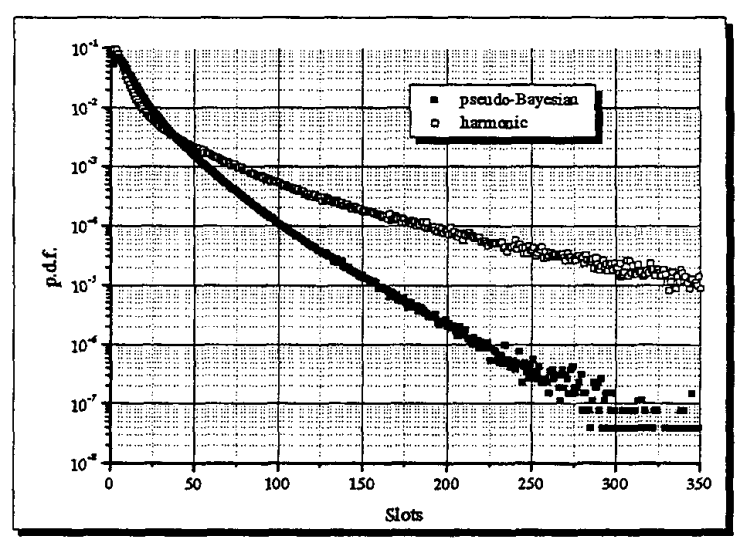

Figure 6 p.d.f of the system delay

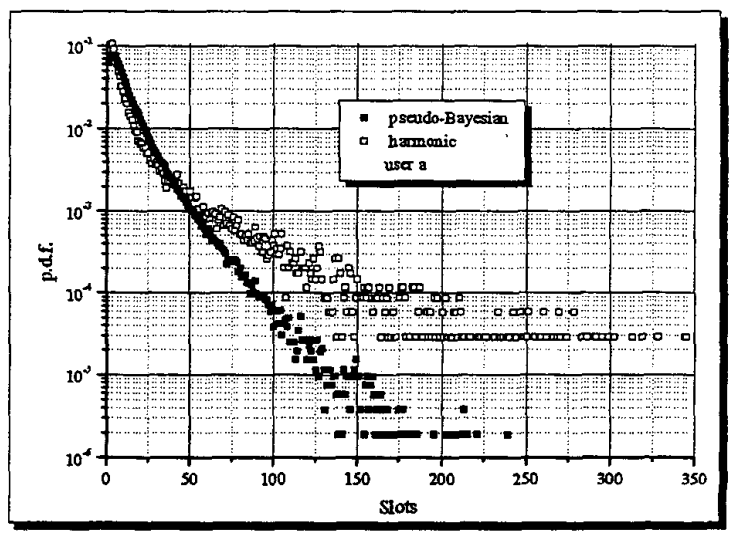

Figure 7 p.d.f of the delay for the user a

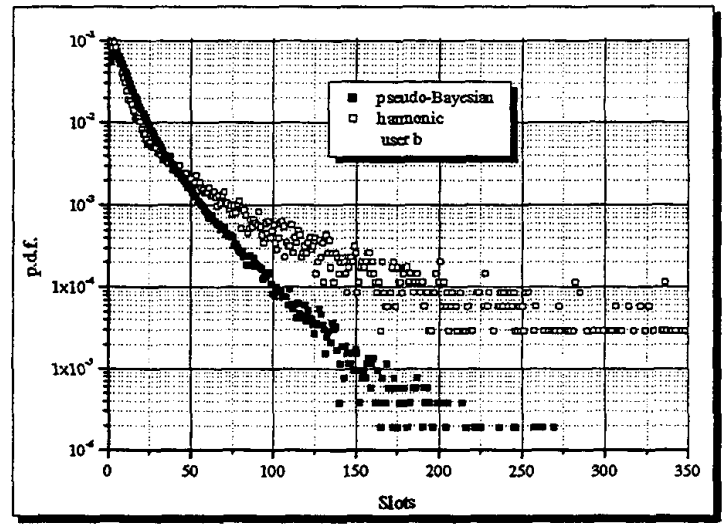

Figure 8 p.d.f of the delay for the user b 


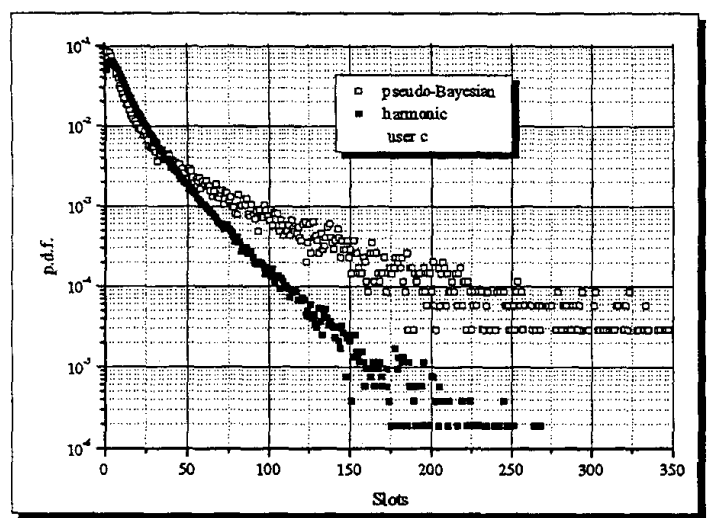

Figure 9 p.d.f of the delay for the user $c$

\section{Conclusion}

The performance of the DQRUMA MAC protocol in a realistic indoor environment has been analysed in this paper.

The efficiency of the pseudo-bayesian as back-off algorithm in the request access phase of the DQRUMA in comparison with efficiency of the harmonic back-off has been evaluated. In particular, plots for the mean delay and the CLR versus the throughput have been presented.

The simulations results indicate that using pseudobayesian, lower CLR and mean delay are obtained. High transmission events with lower probability are also observed when this algorithm is applied.

Consequently, pseudo-bayesian is an adequate back-off algorithm for the DQRUMA.

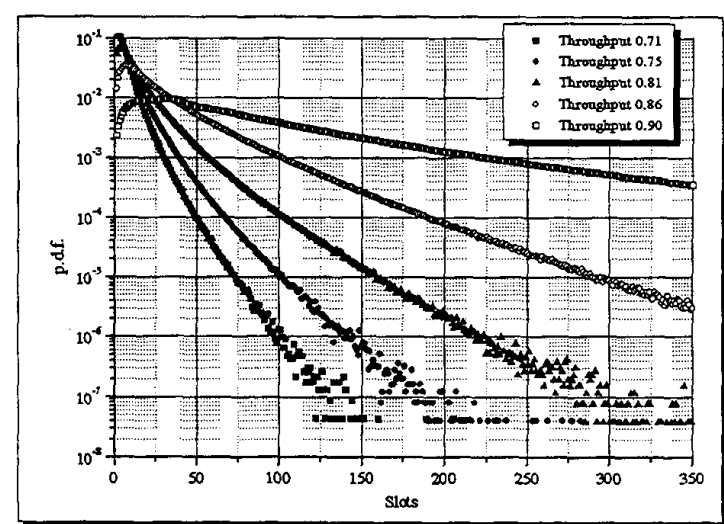

Figure 10 p.d.f of the system delay for several throughput values

\section{References}

[1] D.Raychaudhuri and N.D.Wilson, "ATM-Based transport architecture for multiservices wireless personal communication networks", IEEE JSAC, vol.12, pp. 14011414, October 1994.

[2] M.J.Karol, Z. Liuand K.Y.Eng, 'Distributed-Queuing Request Update Multiple Access (DQRUMA) for Wireless Packet (ATM) Networks," Proc. IEEE INFOCOM'95, pp. 1224-1231.

[3] Ch.V.Verikoukis, J.J.Olmos, "An Efficient Type-II Hybrid ARQ protocol using Punctured R-S Codes for Wireless ATM Networks," Proc. VTC'99-Fall, pp.1725-1729.

[4] L.R.Rabiner, "A Tutorial on Hidden Markov Models and Selected Applications in Speech Recognition", Proc. of the IEEE, Vol. 77, No.2, February 1989.

[5] C.Rapp, "Effects of HPA-Nonlinearity on a 4DPSK/OFDM Signal for Digital Sound Broadcasting System", Proc. of Second European Conference on Satellite Communications, Liège, Belgium, October 22-24, 1991, pp.179-184.

[6] COST 207, Digital land mobile radio communications, Final report, ECSC-EEC-EAEC, Brussels, ISBN 92-8259946-9.

[7] C.M.Dearlove, "A Channel Error Model for the Simulation of Error Control Coding in a Digital Communications System", RMTP/RB/L177, MEC025, June 1991.

[8] R. Rivest, Network Control by Bayesian Broadcast (Report MIT/LCS/TM-285). Cambridge, MA:MIT, Laboratory for computer science.

[9] N. I. Tsiklikis, "Analysis of a Multiaccess Control Scheme," IEEE Trans. Automat. Control, AC-12:10171020. 